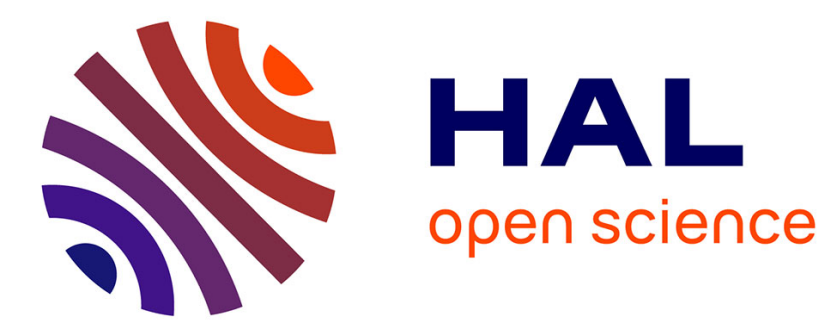

\title{
Fourier series approximation of linear fractional stable motion
}

\author{
Hermine Biermé, Hans-Peter Scheffler
}

\section{To cite this version:}

Hermine Biermé, Hans-Peter Scheffler. Fourier series approximation of linear fractional stable motion. Journal of Fourier Analysis and Applications, 2008, 14, pp.180-202. hal-00178337

\section{HAL Id: hal-00178337 \\ https://hal.science/hal-00178337}

Submitted on 10 Oct 2007

HAL is a multi-disciplinary open access archive for the deposit and dissemination of scientific research documents, whether they are published or not. The documents may come from teaching and research institutions in France or abroad, or from public or private research centers.
L'archive ouverte pluridisciplinaire HAL, est destinée au dépôt et à la diffusion de documents scientifiques de niveau recherche, publiés ou non, émanant des établissements d'enseignement et de recherche français ou étrangers, des laboratoires publics ou privés. 


\title{
FOURIER SERIES APPROXIMATION OF LINEAR FRACTIONAL STABLE MOTION
}

\author{
HERMINE BIERMÉ AND HANS-PETER SCHEFFLER
}

\begin{abstract}
An approximation of the linear fractional stable motion by a Fourier sum is presented. In the continuous sample path case precise error bounds are derived. This approximation method is used to develop a simulation method of the sample path of linear fractional stable motions.
\end{abstract}

\section{INTRODUCTION}

Irregular phenomena appear in various fields of scientific research: traffic volume in modern communication and computer networks and mathematical finance for example. See e.g. $[9,18,11]$ for network traffic and the collection of papers in [5], part B. Important features often discovered are heavy tails, statistical self-similarity and long range dependence. See [4] and [5] for a recent overview. Mathematical models both easy to use and relevant for these applications are fractional stable motions, most prominently the fractional Brownian motion. See [14] for a comprehensive introduction to these processes.

It is a common feature of fractional stable motions that they are self-similar. Moreover they can exhibit long range dependence as well as continuous sample path even in the heavy tailed ( $\alpha$-stable) case. See e.g. [4] and the references therein.

Fix any $0<\alpha \leq 2$ and let $Z_{\alpha}(d z)$ be an independently scattered symmetric $\alpha$ stable $(S \alpha S)$ random measure on $\mathbb{R}$ with Lebesgue control measure $d s$ in the sense of [14]. For $a, b \in \mathbb{R}$ not both equal to zero, $0<H<1$ and $t \in \mathbb{R}$ define

$$
X(t)=X_{a, b}(t)=\int_{\mathbb{R}} g_{a, b}(t, z) Z_{\alpha}(d z)
$$

where

$$
g_{a, b}(t, z)=a\left((t-z)_{+}^{H-1 / \alpha}-(-z)_{+}^{H-1 / \alpha}\right)+b\left((t-z)_{-}^{H-1 / \alpha}-(-z)_{-}^{H-1 / \alpha}\right) .
$$

${ }^{1} 2000$ Mathematics Subject Classification. Primary: 60G18, 60E02; Secondary: 65C50, 68U20. Date: October 10, 2007.

Key words and phrases. fractional stable motion, self-similar process.

Partially supported by NSF grant DMS-0417869. 
The process $\{X(t)\}_{t \in \mathbb{R}}$ is called a linear fractional stable motion (LFSM). Note that the integral is well defined since the integrand is in $L^{\alpha}(\mathbb{R}, d z)$, see e.g. [14]. Also note that if $a=b \neq 0$, then (1.1) reduces to

$$
X(t)=a \int_{-\infty}^{\infty}\left(|t-z|^{H-1 / \alpha}-|z|^{H-1 / \alpha}\right) Z_{\alpha}(d z)
$$

the so-called well balanced linear fractional stable motion.

It follows from basic properties of the random integral definition that $\{X(t)\}_{t \in \mathbb{R}}$ is self-similar with Hurst-index $H$, that is

$$
\{X(c t)\}_{t \in \mathbb{R}} \stackrel{f . d .}{=}\left\{c^{H} X(t)\right\}_{t \in \mathbb{R}}
$$

and has stationary increments, so that for any $h>0$

$$
\{X(t+h)-X(h)\}_{t \in \mathbb{R}} \stackrel{f . d .}{=}\{X(t)\}_{t \in \mathbb{R}} .
$$

Here $\stackrel{f . d .}{=}$ denotes equality in distribution of all finite dimensional marginals.

It follows from Kolmogoroff's Theorem (see [7], Theorem 3.23), using the fact that $\{X(t)\}_{t \in \mathbb{R}}$ has stationary increments and is self-similar with Hurst-coefficient $H$, that if $H>1 / \alpha$ there exists a modification of $\{X(t)\}_{t \in \mathbb{R}}$ with locally Hölder-continuous sample path of order $0<\beta<H-1 / \alpha$ see [17] and [10] for an improvement. We will always choose this version. Note that $H>1 / \alpha$ necessarily implies $1<\alpha \leq 2$. Moreover, the case $H>1 / \alpha$ is also considered the so-called long range dependent case of LFSM, see e.g. [14], Remark on p. 345. We will restrict the major part of this paper to this most important case.

The purpose of this paper is to present a Fourier series approximation of the LSFM $\{X(t)\}_{t \in \mathbb{R}}$ which can be used to efficiently simulate its sample paths. Since there is only a satisfying theory of Fourier series for functions in $L^{p}$ with $p>1$ we will restrict ourself to the case $1<\alpha \leq 2$. To our knowledge, in the heavy tailed case $0<\alpha<2$, there exist three other methods to simulate the sample path of LFSM. The first method, presented in [15], is an effective implementation of the approximation method presented in [14] using the fast-Fourier-transform algorithm. This simulation method generates the so-called fractional stable noise $Y_{n}=X(n)-X(n-1), n=1,2, \ldots$, a stationary sequence, by a Riemann sum approximation of the integral representation. Hence to generate a LFSM sample path of size $N$ one has to compute the cumulative sums $X(n)=\sum_{j=1}^{n} Y_{j}$, for $n=1, \ldots, N$, with an error (in terms of the scale parameter of 
a $S \alpha S$ random variable) growing linearly in $n$. See Corollary 2.1 in [15]. The second method, presented in [19] is based on a limit theorem for sums of moving averages. It generates an approximation of the LFSM by the normalization of the cumulative sums of a linear process. The error in terms of the scale parameter of a $S \alpha S$ random variable is uniform on any compact set. However this method always approximates the LFSM $\{X(t)\}_{t \in \mathbb{R}}$ by a stepwise constant function, see Proposition 1 in [19], even in the continuous sample path case $H>1 / \alpha$. The last method is presented in [2] in a much more general context. It is based on the approximation of the LFSM by the sum of a shot noise series and a fractional Brownian motion, a Gaussian process (see section 6.3). A fast and exact synthesis method for 1-dimensional fractional Brownian motion is known [12], such that this part can be efficiently simulated. However the error due to this approximation is only given in terms of Berry-Essen bounds and can not be compared with the rates of convergence of the shot noise series obtained almost surely and in $L^{r}$ norm.

In contrast to [19], our method will produce directly an approximation of a sample path of LFSM by a continuous function which can be efficiently evaluated at any time point. In particular, we obtain a sample path of a LFSM discretized on a regular grid without additional cost. Moreover, we provide detailed error bounds, in terms of the scale parameter of a $S \alpha S$ random variable, of the approximation, which do not depend on the time point over a compact set, contrary to [15]. Therefore, our approximation can also be used to approximate integral functionals of LFSM. Furthermore our method can be generalized to simulate more general processes than LFSM, obtained through a moving-average integral representation.

This paper is organized as follows. In section 2 we present the general idea behind our method as well as a general convergence result for one fixed time point for any $0<H<1$ and $1<\alpha \leq 2$. In section 3 we consider the continuous sample path case $H>1 / \alpha$. Detailed error estimates in terms of the scale parameter of a $S \alpha S$ random variable (or the standard deviation if $\alpha=2$ ) as well as the convergence of all finite dimensional marginal distributions are obtained in this most important case. We conclude this paper with a description of the simulation algorithm in section 4 . The proofs of Proposition 3.1 and Theorem 3.2 are postponed to the Appendix. 


\section{The APPRoximation Method}

In this section we will describe the general idea of our approximation method for LFSM in the case $0<H<1$ and $1<\alpha \leq 2$ using Fourier-series. Our method is based on the following basic fact of stable integrals. See [14], Chapter 3 for details.

Property (C): For $f_{n}, f \in L^{\alpha}(\mathbb{R}, d z)$ we have

$$
\int_{\mathbb{R}} f_{n}(z) Z_{\alpha}(d z) \rightarrow \int_{\mathbb{R}} f(z) Z_{\alpha}(d z) \quad \text { as } n \rightarrow \infty
$$

in probability, if and only if

$$
\int_{\mathbb{R}}\left|f_{n}(z)-f(z)\right|^{\alpha} d z \rightarrow 0 \quad \text { as } n \rightarrow \infty .
$$

Note that since $\alpha>1$ we know that $\|f\|_{\alpha}=\left(\int_{\mathbb{R}}|f(z)|^{\alpha} d z\right)^{1 / \alpha}$ is a norm on $L^{\alpha}(\mathbb{R}, d z)$ and hence $(2.2)$ is just $\left\|f_{n}-f\right\|_{\alpha} \rightarrow 0$ as $n \rightarrow \infty$.

In the following we will only consider the case $a=1$ and $b=0$ in (1.1). The general case as well as the well-balanced case (1.2) can be dealt with similarly. For any $t \in \mathbb{R}$ fixed, note that since $0<H<1$ the function $z \mapsto(t-z)_{+}^{H-1 / \alpha}-(-z)_{+}^{H-1 / \alpha}$ belongs to $L^{\alpha}(\mathbb{R}, d z)$. Then dominated convergence together with Property (C) implies

$$
Y_{A}(t)=\int_{-A}^{A}\left((t-z)_{+}^{H-1 / \alpha}-(-z)_{+}^{H-1 / \alpha}\right) Z_{\alpha}(d z) \rightarrow X(t) \quad \text { as } A \rightarrow \infty
$$

in probability.

Now fix some (large) $A>0$ and define

$$
e_{k}(z)=\exp \left(i \pi \frac{k}{A} z\right), k \in \mathbb{Z}
$$

Then $\left\{e_{k}(z): k \in \mathbb{Z}\right\}$ is an orthonormal basis in $L^{2}\left([-A, A],(2 A)^{-1} d z\right)$. For a fixed $t \in \mathbb{R}$ let

$$
\rho_{t}(z)=(t-z)_{+}^{H-1 / \alpha}-(-z)_{+}^{H-1 / \alpha} \text { and } \varphi(z)=(-z)_{+}^{H-1 / \alpha}
$$

for $|z| \leq A$ and extend both $\rho_{t}(z)$ and $\varphi(z)$ periodically to $\mathbb{R}$ with period $2 A$, that is $\rho_{t}(z+2 A)=\rho_{t}(z)$ and $\varphi(z+2 A)=\varphi(z)$. Note that $Y_{A}(t)=\int_{-A}^{A} \rho_{t}(z) Z_{\alpha}(d z)$. Since $H>0$ we have $\varphi, \rho_{t} \in L^{\alpha}([-A, A], d s) \subset L^{1}([-A, A], d s)$ since $\alpha>1$. Hence we can define the Fourier-coefficients of $\rho_{t}$ for $k \in \mathbb{Z}$ as

$$
\hat{\rho}_{t}(k)=\frac{1}{2 A} \int_{-A}^{A} \rho_{t}(z) \overline{e_{k}(z)} d z=\left(e^{-i \pi \frac{k}{A} t}-1\right) \hat{\varphi}(k),
$$


where $\hat{\varphi}(k)=\frac{1}{2 A} \int_{-A}^{A} \varphi(z) \overline{e_{k}(z)} d z$. Since $1<\alpha \leq 2$, using the fact that the Fourierseries of an $L^{\alpha}\left([-A, A],(2 A)^{-1} d z\right)$-function $f$ converges to $f$ in $L^{\alpha}\left([-A, A],(2 A)^{-1} d z\right)$ (see e.g. [8], Theorem on p. 50), we have

$$
\sum_{k=-M}^{M} \hat{\rho}_{t}(k) e_{k}(z) \rightarrow \rho_{t}(z) \quad \text { as } M \rightarrow \infty
$$

in $L^{\alpha}\left([-A, A],(2 A)^{-1} d z\right)$. Therefore, using Property $(\mathrm{C})$ again, we conclude

$$
Y_{A, M}(t)=\int_{-A}^{A}\left(\sum_{k=-M}^{M} \hat{\rho}_{t}(k) e_{k}(z)\right) Z_{\alpha}(d z) \rightarrow Y_{A}(t) \quad \text { as } M \rightarrow \infty
$$

in probability. Note that

$$
Y_{A, M}(t)=\sum_{k=-M}^{M} \hat{\rho}_{t}(k) \int_{-A}^{A} e_{k}(z) Z_{\alpha}(d z) .
$$

We now approximate $\int_{-A}^{A} e_{k}(z) Z_{\alpha}(d z)$ by an analogue of a Riemann sum. For integers $L \geq 1$ let $z_{j}=z_{j}(A, L)=j(A / L)$ for $j=-L, \ldots, L$ and let $\Delta z_{j}=\left[z_{j}, z_{j+1}[\right.$. Observe that since $e_{k}(z)$ is uniformly continuous on $[-A, A]$ we have

$$
\sum_{j=-L}^{L-1} e_{k}\left(z_{j}\right) 1_{\Delta z_{j}}(z) \rightarrow e_{k}(z) \quad \text { as } L \rightarrow \infty
$$

uniformly on $[-A, A]$ and hence in $L^{\alpha}\left([-A, A],(2 A)^{-1} d z\right)$. Therefore, in view of Property (C) we get

$$
\hat{Z}_{A, L}(k)=\sum_{j=-L}^{L-1} e_{k}\left(z_{j}\right) Z_{\alpha}\left(\Delta z_{j}\right) \rightarrow \int_{-A}^{A} e_{k}(z) Z_{\alpha}(d z) \quad \text { as } L \rightarrow \infty
$$

in probability. Note that $Z_{\alpha}\left(\Delta z_{j}\right), j=-L, \ldots, L-1$ are i.i.d. $S \alpha S$ random variables with scale $(A / L)^{1 / \alpha}$, since by normalization we assume that the scale of $Z_{\alpha}([0,1])$ equals one.

We now define

$$
Y_{A, M, L}(t)=\sum_{k=-M}^{M} \hat{\rho}_{t}(k) \hat{Z}_{A, L}(k)=\sum_{k=-M}^{M}\left(e^{-i \pi \frac{k}{A} t}-1\right) \hat{\varphi}(k) \hat{Z}_{A, L}(k)
$$

to be our approximation of the LFSM $X(t)$ for any $t \in \mathbb{R}$ fixed. In view of $(2.3)-(2.6)$ we haven proven: 
Theorem 2.1. If $1<\alpha \leq 2$ we have, for any fixed $t \in \mathbb{R}$ that

$$
\begin{aligned}
\lim _{L \rightarrow \infty} Y_{A, M, L}(t) & =Y_{A, M}(t) \\
\lim _{M \rightarrow \infty} Y_{A, M}(t) & =Y_{A}(t)
\end{aligned}
$$

and

$$
\lim _{A \rightarrow \infty} Y_{A}(t)=X(t)
$$

in probability.

Observe that

$$
\hat{Z}_{A, L}(k)=\sum_{j=-L}^{L-1} e^{2 i \pi \frac{j k}{2 L}} Z_{\alpha}\left(\Delta z_{j}\right)
$$

can be viewed as the discrete Fourier-transform of the sequence $\left(Z_{\alpha}\left(\Delta z_{j}\right): j=\right.$ $-L, \ldots, L-1)$. Hence we choose $L>M$ a power of 2 in our approximation method in order to apply the Fast-Fourier-Transform algorithm to compute $\widehat{Z}_{A, L}(k)$ for $-M \leq k \leq M$ from a generated sequence $Z_{\alpha}\left(\Delta z_{j}\right)$ of i.i.d. $S \alpha S$ random variables. However, the derivation of Theorem 2.1 does not allow directly to have $M, L \rightarrow \infty$ simultaneously. In the next section we will show that if $H>1 / \alpha$ our method also works if $L=M+1$ or $L=2 M \rightarrow \infty$, see Corollary 3.5 and Remark 3.6 below. Moreover, we improve Theorem 2.1 by providing error bounds and show convergence of all finite-dimensional marginal distributions.

\section{ERror estimates}

In this section we derive error estimates of our approximations $\left\{Y_{A, M, L}(t)\right\}_{t \in \mathbb{R}}$ to $\left\{Y_{A}(t)\right\}_{t \in \mathbb{R}}$ and of the latter to the LFSM $\{X(t)\}_{t \in \mathbb{R}}$ in terms of the scale parameter $\|\xi\|_{\alpha}$ of a $S \alpha S$ random variable $\xi$, where the notation $\|\cdot\|_{\alpha}$ is used according to the situation for a function or a $S \alpha S$ random variable. Recall from [14] that a $S \alpha S$ random variable $\xi$ has the characteristic function $\mathbb{E}\left(e^{i x \xi}\right)=\exp \left(-C_{\alpha}^{\alpha}|x|^{\alpha}\right)$, where $C_{\alpha}$ denotes the scale parameter of $\xi$. We also use the notation $C_{\alpha}=\|\xi\|_{\alpha}$ which is very illuminating when dealing with stable integrals because the scale parameter of the stable integral $\xi=\int_{\mathbb{R}} g(z) Z_{\alpha}(d z)$ is $\|\xi\|_{\alpha}=C_{\alpha}\left(\int_{\mathbb{R}}|g(z)|^{\alpha} d z\right)^{1 / \alpha}=C_{\alpha}\|g\|_{\alpha}$, where $C_{\alpha}$ is the scale parameter of $Z_{\alpha}([0,1])$. We will assume without loss of generality that $C_{\alpha}=1$. 
In the following we only consider the continuous and long range dependence case $H>1 / \alpha$. Note that by $(2.3)$ we can write

$$
Y_{A}(t)=\int_{-A}^{A} \rho_{t}(z) Z_{\alpha}(d z)
$$

where $\rho_{t}$ is a $2 A$-periodic function which is now bounded and continuous. In fact $\rho_{t}(z)=\varphi(t-z)-\varphi(-z)$ where $\varphi(z)=a(z)_{+}^{H-1 / \alpha}+b(z)_{-}^{H-1 / \alpha}$ for $z \in[-A, A[$. As before we will only consider the case $a=1$ and $b=0$ as well as the well balanced case $a=b=1$ which will allow better estimates because of the symmetry of $\varphi(z)=$ $|z|^{H-1 / \alpha}$ in this particular case.

We first analyze the effect of truncation on the integral defining $X(t)$ as in (2.3) above. We fix any $0<T<A$ and consider the processes only on the finite interval $|t| \leq T$. The rate of convergence is obtained using the mean value theorem and the decreasing order of the kernel function $\varphi$.

Proposition 3.1. Assume $1<\alpha \leq 2$ and $1 / \alpha<H<1$. Then, for all $T>0$ and $A>0$ we have for all $|t| \leq T$,

$$
\left\|X(t)-Y_{A}(t)\right\|_{\alpha} \leq C_{1}(H, \alpha) T(A-T)^{-(1-H)},
$$

where $C_{1}(H, \alpha)=2(H-1 / \alpha)(\alpha(1-H))^{-1 / \alpha}$.

Proof. See the Appendix.

We now present the main result of this section. The following theorem provides error bounds in terms of the scale parameter of our Fourier series approximation $Y_{A, M, L}(t)$ of $Y_{A}(t)$. Note that by (2.6) and (2.7) we can write

$$
Y_{A, M, L}(t)=\sum_{j=-L}^{L-1}\left(\sum_{k=-M}^{M} \hat{\rho}_{t}(k) e_{k}\left(z_{j}\right)\right) \int_{z_{j}}^{z_{j+1}} Z_{\alpha}(d y) .
$$

Recall from [8] that, for $2 A$ periodic functions the Dirichlet kernel is given by

$$
D_{A, M}(x)=\sum_{k=-M}^{M} e^{i \pi \frac{k}{A} x}=\frac{\sin ((2 M+1) \pi x / 2 A)}{\sin (\pi x / 2 A)}
$$

and that for integrable $2 A$-periodic functions $f$ we have

$$
\sum_{k=-M}^{M} \widehat{f}(k) e_{k}(x)=D_{A, M} * f(x)
$$


where for integrable $2 A$-periodic functions $f, g$ the convolution $f * g$ is defined as

$$
f * g(x)=\frac{1}{2 A} \int_{-A}^{A} f(x-z) g(z) d z
$$

and

$$
\widehat{f}(k)=\frac{1}{2 A} \int_{-A}^{A} f(z) \overline{e_{k}(z)} d z
$$

Therefore we get

$$
\sum_{k=-M}^{M} \widehat{\rho}_{t}(k) e_{k}(x)=D_{A, M} * \rho_{t}(x) .
$$

Hence we can rewrite (3.2) as

$$
Y_{A, M, L}(t)=\sum_{j=-L}^{L-1}\left(D_{A, M} * \rho_{t}\right)\left(z_{j}\right) \int_{z_{j}}^{z_{j+1}} Z_{\alpha}(d y) .
$$

Theorem 3.2. Assume $1<\alpha \leq 2$ and $1 / \alpha<H<1$. Then, for all $T, A>0$, $M \geq e^{4}$ and $L>M$, for any $|t| \leq T$

$$
\left\|Y_{A}(t)-Y_{A, M, L}(t)\right\|_{\alpha} \leq \varepsilon_{\varphi}(A, M, L),
$$

where, if $\varphi(z)=(-z)_{+}^{H-1 / \alpha}$ (or if $\varphi(z)=(-z)_{-}^{H-1 / \alpha}$ )

$$
\varepsilon_{\varphi}(A, M, L)=A^{H}\left(C_{3}(H, \alpha) M^{-1 / 2}+C_{4}(H, \alpha) L^{-1 / \alpha} \log (M)\right),
$$

with $C_{3}(H, \alpha)=2^{3 / 2+1 / \alpha}(2+H-1 / \alpha)$ and, if $\varphi(z)=|z|^{H-1 / \alpha}$

$$
\varepsilon_{\varphi}(A, M, L)=A^{H}\left(C_{3}(H, \alpha) M^{-1 / 2-(H-1 / \alpha)}+C_{4}(H, \alpha) L^{-1 / \alpha} \log (M)\right),
$$

with $C_{3}(H, \alpha)=2^{3 / 2+1 / \alpha}(1+2 H-2 / \alpha)^{-1 / 2}(1+H-1 / \alpha)$.

In both cases we have $C_{4}(H, \alpha)=2^{3}\left(2^{1+H-1 / \alpha}(\alpha+1)^{-1 / \alpha}+1\right)$.

Sketch of the proof. Since $\rho_{t} \in L^{\alpha}\left([-A, A],(2 A)^{-1} d x\right)$ with $\alpha>1$, we know from the theorem on page 50 of [8] that $\rho_{t}(\cdot)=\sum_{k=-\infty}^{+\infty} \hat{\rho}_{t}(k) e_{k}(\cdot)$, where the series converges in $L^{\alpha}$. Furthermore,

$$
\rho_{t}=\sum_{|k| \leq M} \hat{\rho}_{t}(k) e_{k}+\sum_{|k|>M} \hat{\rho}_{t}(k) e_{k}=D_{A, M} * \rho_{t}+\sum_{|k|>M} \hat{\rho}_{t}(k) e_{k}
$$

Since $\alpha>1$, we can use Minkowski's inequality to get

$$
\left\|Y_{A}(t)-Y_{A, M, L}(t)\right\|_{\alpha} \leq V(A, M)+U(A, M, L),
$$


with, on the one hand

$$
V(A, M)=\left(\int_{-A}^{A}\left|\sum_{|k|>M} \hat{\rho}_{t}(k) e_{k}(y)\right|^{\alpha} d y\right)^{1 / \alpha}
$$

which we bound by $C_{3}(H, \alpha) A^{H} M^{-1 / 2}$, using the rate of convergence of $\sum_{|k|>M}\left|\hat{\rho}_{t}(k)\right|^{2}$, Hölder's inequality and Plancherel-identity. On the other hand

$$
U(A, M, L)=\left(\int_{-A}^{A}\left|\sum_{j=-L}^{L-1} 1_{\left[z_{j}, z_{j+1}[\right.}(y)\left[D_{A, M} * \rho_{t}(y)-D_{A, M} * \rho_{t}\left(z_{j}\right)\right]\right|^{\alpha} d y\right)^{1 / \alpha}
$$

is bounded by $C_{4}(H, \alpha) A^{H} L^{-1 / \alpha} \log (M)$, using the mean value theorem, Hölder regularity of order $H-\frac{1}{\alpha}$ of the function $\rho_{t}$ and the fact that $\frac{1}{2 A} \int_{-A}^{A}\left|D_{A, M}(x)\right| d x \leq$ $2 \log (M)$.

Combining Proposition 3.1 and Theorem 3.2 we get

Corollary 3.3. Assume $1<\alpha \leq 2$ and $1 / \alpha<H<1$. Then, for all $T, A>0$, $M \geq e^{4}$ and $L>M$, for any $|t| \leq T$

$$
\left\|X(t)-Y_{A, M, L}(t)\right\|_{\alpha} \leq C_{1}(H, \alpha) T(A-T)^{H-1}+\varepsilon_{\varphi}(A, M, L),
$$

where $\varepsilon_{\varphi}$ is given by (3.4) or (3.5) according to $\varphi$ and $C_{1}(H, \alpha)=2(H-$ $1 / \alpha)(\alpha(1-H))^{-1 / \alpha}$.

Observe that by Theorem 3.2 it follows that we can choose $L=M+1$ in (2.7) and still get convergence.

Remark 3.4. Let us point out that the error bound estimate does not depend on the time point $t$. Therefore, one advantage of this method is that integral functionals of the LFSM can be approximated efficiently just as well as values of the process at time points. Actually one can consider the integral $\int_{-T}^{T} g(t) X(t) d t$ for a large class of functions $g$ defined on a compact set $[-T, T]$. Replacing $X(t)$ by its approximation $Y_{A, M, L}(t)$ given by $(2.7)$ this integral can be approximated by

$$
\int_{-T}^{T} g(t) Y_{A, M, L}(t) d t=\sum_{k=-M}^{M}\left(\int_{-T}^{T}\left(e^{-i \pi \frac{k}{A} t}-1\right) g(t) d t\right) \hat{\varphi}(k) \hat{Z}_{A, L}(k) .
$$

Therefore, computing the last deterministic integrals in advance, one can quickly generate approximate realizations of $\int_{-T}^{T} g(t) X(t) d t$. Moreover, since $X(t)-Y_{A, M, L}(t)$ is a $S \alpha S$ random variable with $\alpha>1$, there is a constant $c_{\alpha}(1)$ such that we have 
$\mathbb{E}\left|X(t)-Y_{A, M, L}(t)\right| \leq c_{\alpha}(1) \mid\left\|X(t)-Y_{A, M, L}(t)\right\|_{\alpha}$, according to Property 1.2 .17 p.18 of [14]. Then

$$
\mathbb{E}\left|\int_{-T}^{T} g(t)\left(X(t)-Y_{A, M, L}(t)\right) d t\right| \leq c_{\alpha}(1) \int_{-T}^{T}|g(t)| \cdot\left\|X(t)-Y_{A, M, L}(t)\right\|_{\alpha} d t,
$$

which gives the rate of convergence of the approximations of $\int_{-T}^{T} g(t) X(t) d t$ by Corollary 3.3.

We obtain the convergence of all finite-dimensional marginal distributions from Corollary 3.3.

Corollary 3.5. Assume $1<\alpha \leq 2$ and $1 / \alpha<H<1$. Then, for all $T>0$, as $A, M, L \rightarrow+\infty$, such that $\varepsilon_{\varphi}(A, M, L) \rightarrow 0$ where $\varepsilon_{\varphi}$ is given by (3.4) or (3.5) according to $\varphi$, the approximations $\left\{Y_{A, M, L}(t)\right\}_{t \in[-T, T]}$ converge in finite-dimensional marginal distributions to the LFSM $\{X(t)\}_{t \in[-T, T]}$.

Proof. Fix any $t_{1}, \ldots, t_{n} \in[-T, T]$ and $\lambda_{1}, \ldots, \lambda_{n} \in \mathbb{R}$. Then by Corollary 3.3 and the triangle inequality we obtain

$$
\left\|\sum_{j=1}^{n} \lambda_{j} X\left(t_{j}\right)-\sum_{j=1}^{n} \lambda_{j} Y_{A, M, L}\left(t_{j}\right)\right\|_{\alpha} \leq \sum_{j=1}^{n}\left|\lambda_{j}\right|\left\|X\left(t_{j}\right)-Y_{A, M, L}\left(t_{j}\right)\right\|_{\alpha} \rightarrow 0
$$

as $A, M, L \rightarrow \infty$ and the corresponding condition is fulfilled. Since for a sequence $\xi_{n}$ of $S \alpha S$ random variable $\left\|\xi_{n}\right\|_{\alpha} \rightarrow 0$ implies $\xi_{n} \rightarrow 0$ in probability and the above relation holds for all $\lambda_{1}, \ldots, \lambda_{n} \in \mathbb{R}$, the convergence of all finite-dimensional marginal distributions follows.

Remark 3.6. If we let $L=M+1$ and $A=A(M)=M^{\rho}$ for some $0<\rho<1 / 2$, since $\varepsilon_{\varphi}\left(M^{\rho}, M, M+1\right) \underset{M \rightarrow+\infty}{\longrightarrow} 0$, we get from Corollary 3.5 that $\left\{Y_{M^{\rho}, M, M+1}(t)\right\}_{t \in \mathbb{R}} \Rightarrow$ $\{X(t)\}_{t \in \mathbb{R}}$ as $M \rightarrow \infty$ for all finite-dimensional marginal distributions.

Remark 3.7. In the situation of Corollary 3.5 it is a challenging open problem to achieve tightness and hence convergence in distribution on some suitable function space.

\section{The Simulation Algorithm}

In this section we present an effective simulation algorithm of the sample path of the LFSM $\{X(t)\}_{t \in \mathbb{R}}$ defined by (1.1) based on our Fourier approximation given by 
(2.7). We will only consider the so called well balanced case given by (1.2). Then

$$
Y_{A, M, L}(t)=\sum_{k=-M}^{M}\left(e^{-i \pi \frac{k}{A} t}-1\right) \hat{\varphi}(k) \hat{Z}_{A, L}(k) .
$$

Recall from (2.6) that for $k=-M, \ldots, M$ in this case we have

$$
\hat{Z}_{A, L}(k)=\sum_{j=-L}^{L-1} e^{2 i \pi k j / 2 L} Z_{\alpha}\left(\Delta z_{j}\right)
$$

where $Z_{\alpha}\left(\Delta z_{j}\right), j=-L, \ldots, L-1$ are i.i.d. $S \alpha S$ random variables with scale $(A / L)^{1 / \alpha}$. This sequence can easily and exactly be simulated using the algorithm from Chambers et al., see [1]. Note that (4.2) is the discrete Fourier transform of the vector $\left(Z_{\alpha}\left(\Delta z_{j}\right): j=-L, \ldots, L-1\right)$ and can be effectively computed using a variant of the fast Fourier transform algorithm (FFT). Moreover, since $\varphi(z)=|z|^{H-1 / \alpha}$ we know from Lemma 5.1(b), especially (5.4) that

$$
\hat{\varphi}(k)=A^{H-1 / \alpha} k^{-1-H+1 / \alpha} \int_{0}^{k} v^{H-1 / \alpha} \cos (\pi v) d v .
$$

The integral in (4.3) can be effectively computed by decomposing

$$
\int_{0}^{k} v^{H-1 / \alpha} \cos (\pi v) d v=\sum_{j=1}^{k} \int_{j-1}^{j} v^{H-1 / \alpha} \cos (\pi v) d v
$$

and approximating each summand by Simpson's rule, see e.g. [6], Theorem 2.3. Our simulation study presented below shows that the approximation error resulting from applying Simpson's rule has little effect on the accuracy of our simulation method.

Let us denote

$$
W_{A, M, L}(t)=\sum_{k=-M}^{M} e^{-i \pi \frac{k}{A} t} \hat{\varphi}(k) \hat{Z}_{A, L}(k)
$$

the discrete Fourier transform of the vector $\left(\hat{\varphi}(k) \hat{Z}_{A, L}(k)\right)_{-M \leq k \leq M}$ such that by $(4.1)$

$$
Y_{A, M, L}(t)=W_{A, M, L}(t)-W_{A, M, L}(0) .
$$

Let us point out that the fast Fourier transform algorithm allows to compute $W_{A, M, L}$ and $Y_{A, M, L}$ at any point $t_{l}=\frac{2 A}{2 M+1} l$ for $-M \leq l \leq M$ with $O\left(M \log _{2} M\right)$ operations. Let us assume that we want to generate an approximate sample path of the LFSM $\{X(t)\}_{t \in \mathbb{R}}$ with Hurst index $H$ over an interval $[a, b]$, given by $N+1$ equidistant time points. Since the LFSM has stationary increments we may consider $[0, b-a]$ instead 
of $[a, b]$. Let $\delta_{N}=\frac{b-a}{N}$ be the step size of the time points over $[0, b-a]$. Using the self-similarity of order $H$ of $\{X(t)\}_{t \in \mathbb{R}}$ we have

$$
\left\{X(0), \ldots, X\left(N \delta_{N}\right)\right\} \stackrel{d}{=}\left(\delta_{N} \frac{2 M+1}{2 A}\right)^{H}\left\{X\left(t_{0}\right), \ldots, X\left(t_{N}\right)\right\} .
$$

Therefore we only have to generate $W_{A, M, L}$ at any point $\left(t_{l}\right)_{0 \leq l \leq N-1}$ for some $M \geq N$ which can be done with only $O\left(M \log _{2} M\right)$ operations.

We now formulate our simulation algorithm (A MATLAB code can be obtained from the authors upon request). Given a number $N$ of points, a truncation point $A>0$ and some large integers $M, L \geq N$ (usually one should pick $M=2^{p} \geq N$ and $L=2^{q}$ with $p>q$ in view of the FFT algorithm) we compute:

Step 1: Compute $\hat{\varphi}(k), k=-M, \ldots, M$ by (4.3)

Step 2: Generate $2 L S \alpha S$ random variables $Z_{-L}, \ldots, Z_{L-1}$ and set $\bar{Z}_{j}=(A / L)^{1 / \alpha} Z_{j}$

Step 3: Compute the FFT of $\left(\bar{Z}_{-L}, \ldots, \bar{Z}_{L-1}\right)$ to obtain $(\hat{Z}(-M), \ldots, \hat{Z}(M))$

Step 4: Set $\hat{W}_{k}=\hat{\varphi}(k) \cdot \hat{Z}(k)$ for $k=-M, \ldots, M$

Step 5: Compute the FFT of $\left(\hat{W}_{-M}, \ldots, \hat{W}_{M}\right)$ to get $\left(W_{A, M, L}\left(t_{-M}\right), \ldots, W_{A, M, L}\left(t_{M}\right)\right)$ Step 6: Compute $Y_{A, M, L}\left(t_{l}\right)=W_{A, M, L}\left(t_{l}\right)-W_{A, M, L}(0)$ for $0 \leq l \leq N$.

Note that for any particular sample path, Step 1 of the algorithm have only to be executed once in an initializing step. After the initialization the complexity to compute one approximation sample path is $O\left(M \log _{2}(M)\right)$ when choosing $M$ a power of 2. Let us point out that it is about the same cost than in [15] and [19] to get approximations not of the LFSM itself but of the linear fractional stable noise. Therefore, to obtain approximations of the LFSM, the authors have one more step than us which is to compute cumulative sums of the noise.

Sample paths realizations of LFSM for different $H$ and $\alpha$ are given in Figure 1. The computational time (on a dual core PowerMac G5) for $L=2^{18}$ and $N=M=2^{16}$ is 46 seconds for the initialization steps 1 and 0.7 second for each sample path. Let us point out that, even if error bounds of the approximations have only been proved in the cases $\alpha \in(1,2)$ and $H>1 / \alpha$, one can use the code for any values of $\alpha \in(0,2)$ 

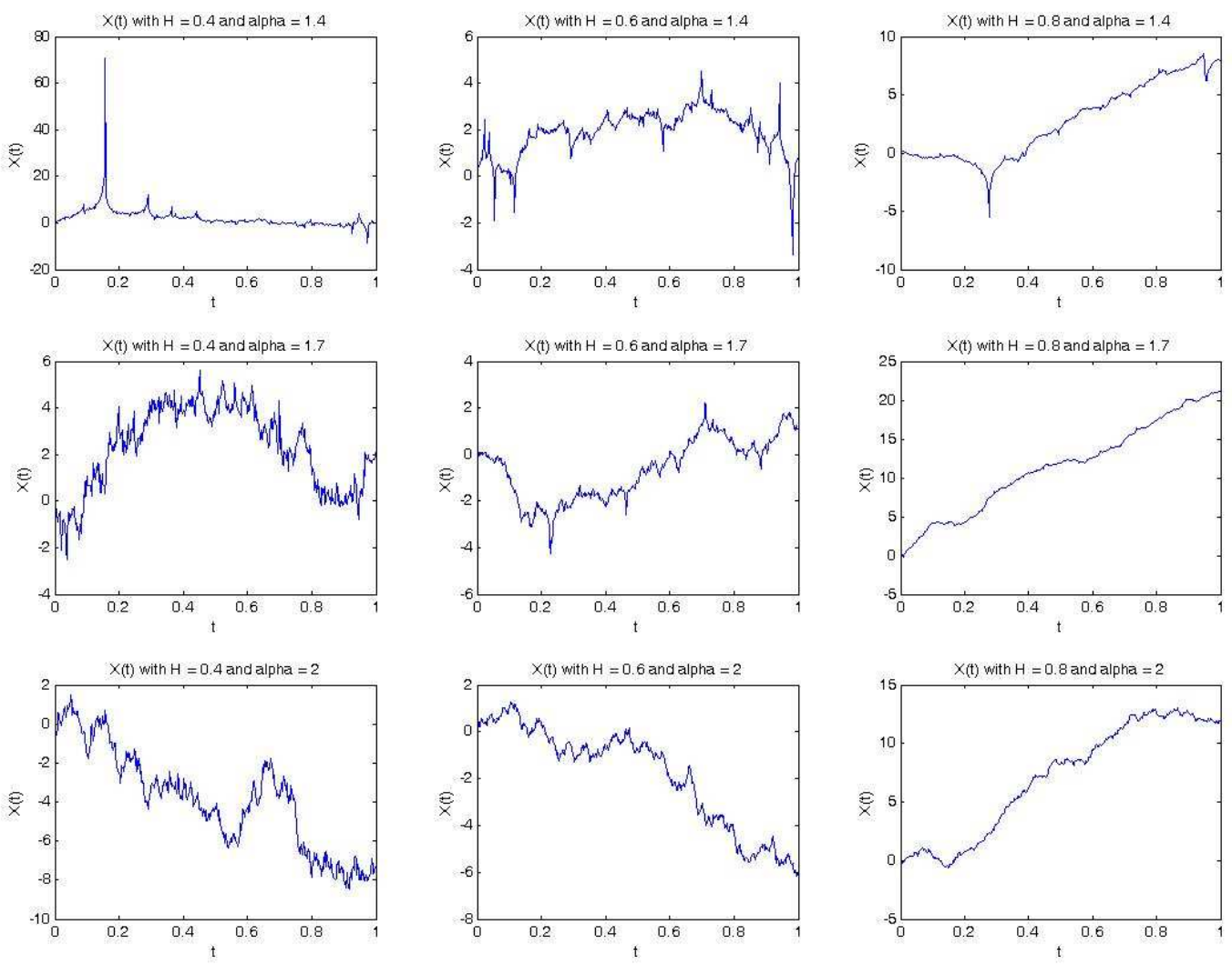

FigURE 1. Approximations of LFSM sample path for various $H, \alpha$. We used $A=101$ and $L=2^{18}$ and $N=M=2^{16}$.

and $H \in(0,1)$. In fact, in view of Theorem 2.1 we still get an approximation. Actually, note that $H>1 / \alpha$ in Figure 1 only for $\alpha=1.4$ and $H=0.8$ or $\alpha \in\{1.7,2\}$ and $H \in\{0.6,0.8\}$. Finally let us mention that when $\alpha=2$ the LFSM is just the well known fractional Brownian motion for which a lot of numerical methods have been proposed these last years. Moreover, in this particular case, there exists a fast and exact synthesis method [12], based on the Choleski decomposition of the covariance function and on the stationarity of the increments that allows to apply the embedding circulant matrix method [3]. Then our approximation is not really relevant for this case. 
To illustrate the quality of the approximation given by this method we estimate the Hurst parameter $H$ of the LFSM using two type of estimators studied in [16], namely the wavelet $\hat{H}_{W T}$ and the FIRT $\hat{H}_{F I R T}$ estimators based on discrete wavelet transforms of the approximation for the first one, and on discrete differences for the second one. The results obtained for a fixed parameter $H=0.7$ with respect to the stability index $\alpha$ are given in Figure 2. It can be compared to those of Figure 4 in [16]. Let us point out that both estimators underestimate the real theoretical value of $H$. However, for $H \geq 1 / \alpha$ the bias of the two estimators is relatively low (about 0.02 ) and the standard deviation is very small (less than $10^{-3}$ ) while in [16] the bias is about 0.01 with standard deviation greater than 0.03 .
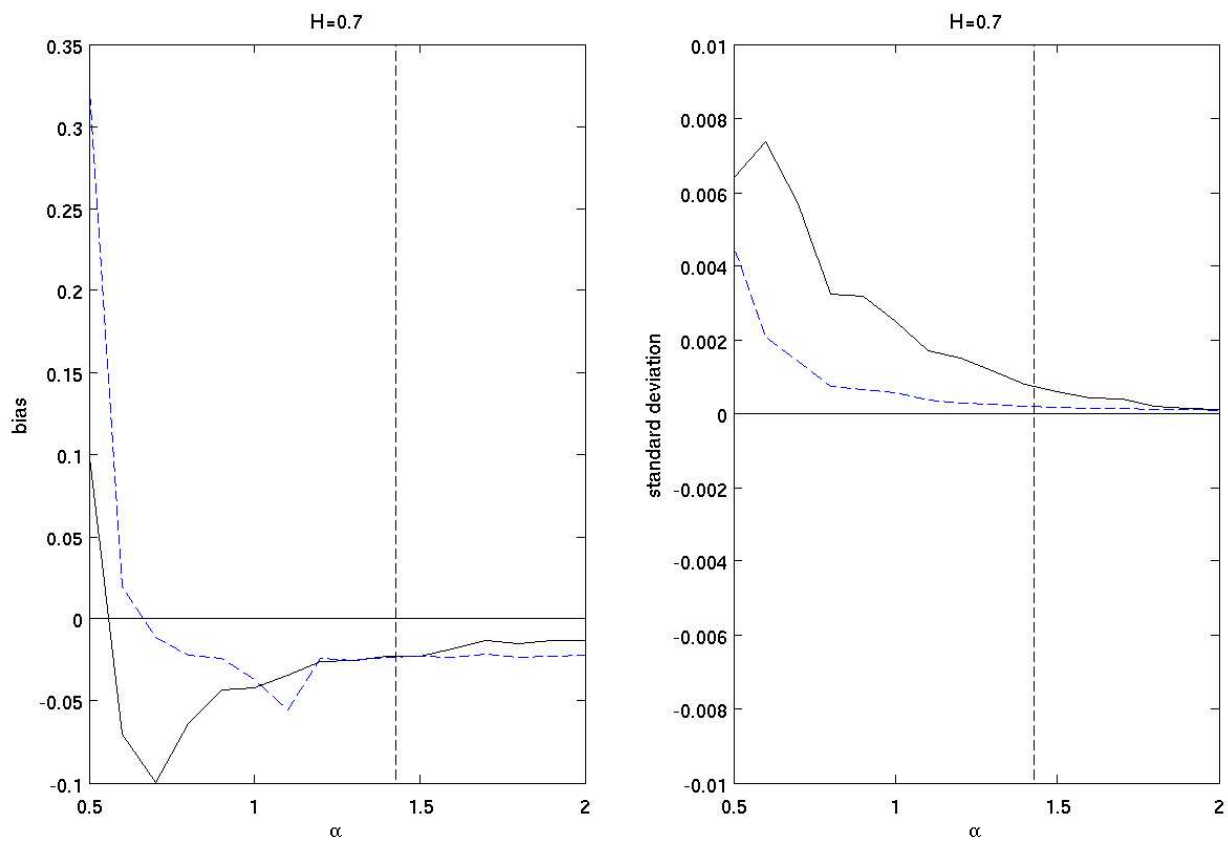

FIGURE 2. Bias and standard deviations of the FIRT estimators $\widehat{H}_{F I R T}-H$ (solid line) and the wavelet estimators $\widehat{H}_{W T}$ (dashed line) studied in [16] as functions of $\alpha$ when $H=0.7$. The broken vertical line located at $\alpha=1 / H$ indicates on the right the long range dependence cases. The bias and standard deviations were computed by using samples of $n=256$ independent replications of the estimators, obtained from 256 independently simulated paths of the LFSM process for $N=M=2^{16}$ points, $L=2^{18}$ and $A=101$. 

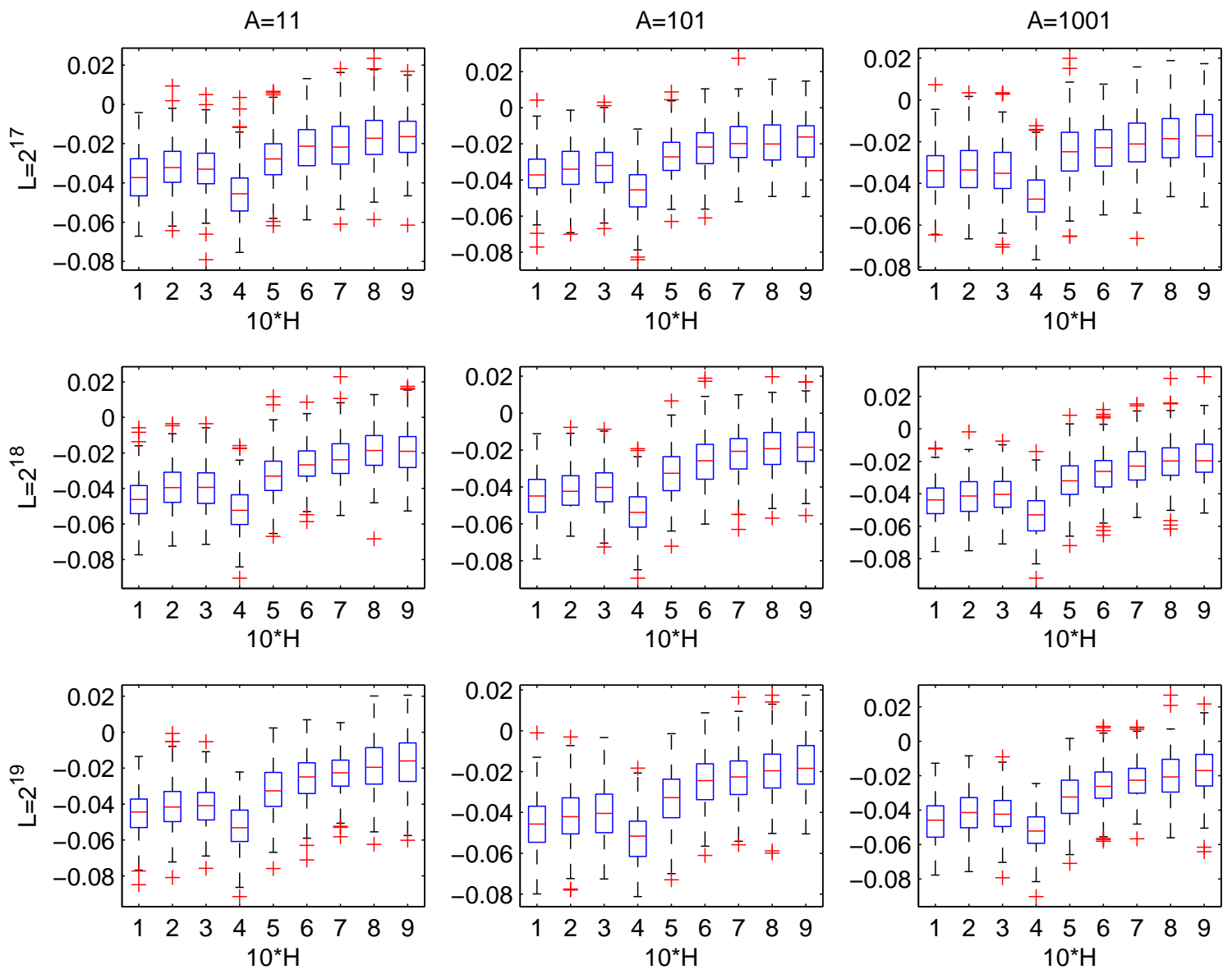

FiguRE 3 . This figure contains Boxplots for the bias $\widehat{H}_{W T}-H$ for the wavelet estimators $\widehat{H}_{W T}$ studied in [16]. As in Figure 3 in [15] these Boxplots were computed by using samples of $n=256$ independent replications of the estimators, obtained from 256 independently simulated paths of the LFSM process for an index of stability $\alpha=1.5$ for $M=2^{16}$ points. The values of $H$ are $H=0.1,0.2, \ldots, 0.9$ (note that $0.6<1 / \alpha<0.7$ such that $H=0.7,0.8$ and 0.9 correspond to long-range dependence). The values of the discretization parameters $A$ and $L$, used in the simulation algorithm are indicated in the margins of the figure.

Figures 3 and 4 contain Boxplots for the bias $\mathbb{E}\left(\hat{H}_{W T}\right)-H$ and $\mathbb{E}\left(\hat{H}_{F I R T}\right)-H$ for a fixed index of stability $\alpha=1.5$. In this simulation study a sample of 256 independent copies of our LFSM approximation of size $N=M=2^{16}$ points were generated using three different values of the truncation parameter $A=11,101$ and 1001, three different values of the step parameter $L=2^{17}, 2^{18}$ and $2^{19}$ for the following values of the Hurst parameter $H=0.1,0.2, \ldots, 0.9$. 
As already noticed in Figure 2 for $H=0.7$, both estimators underestimate the real theoretical value of $H$ for any $H=0.1, \ldots, 0.9$. Let us also point out that the FIRT estimator (Figure 4) exhibits a larger variability than the wavelet one (Figure 3).
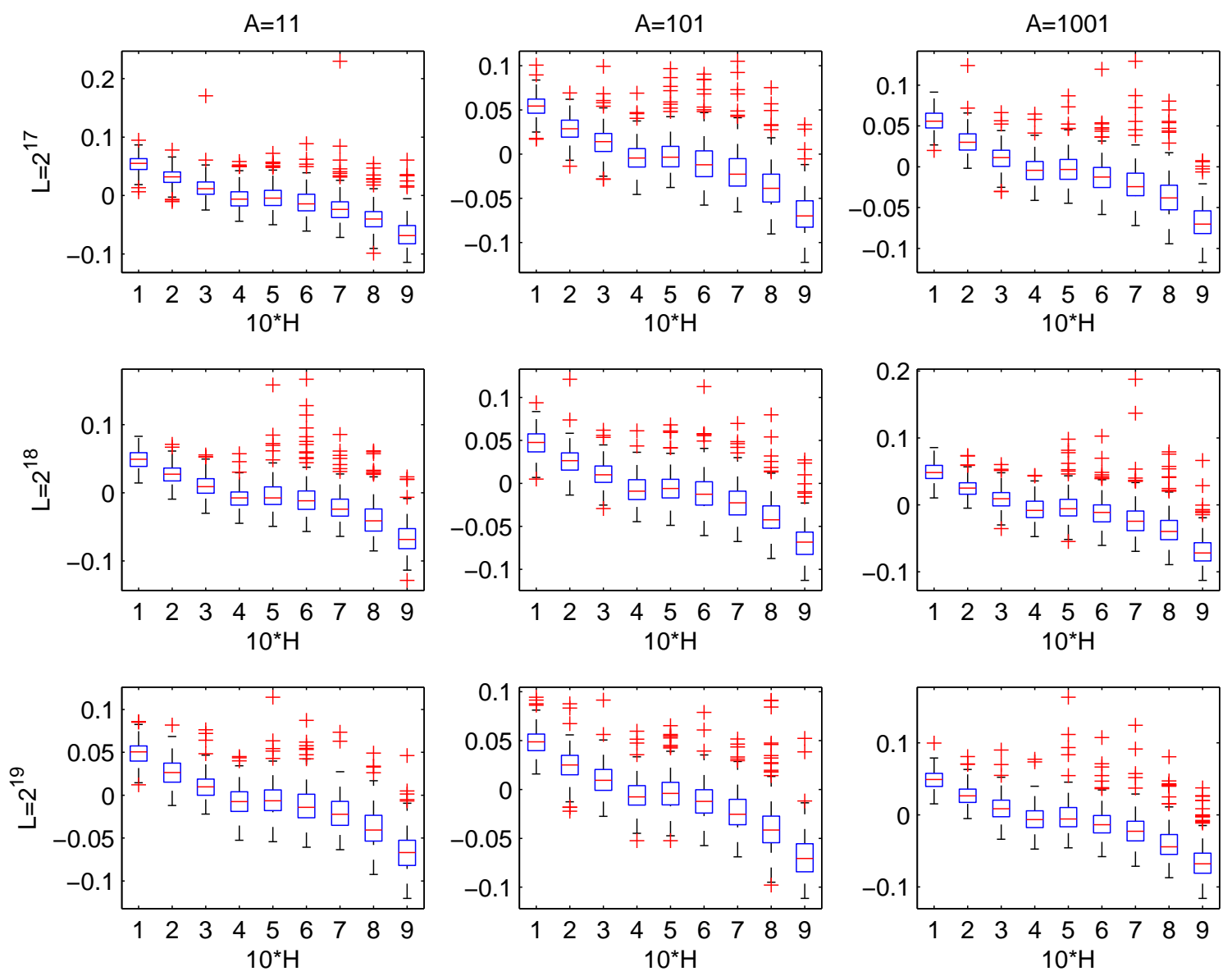

FIgURE 4. This figure contains Boxplots for the bias $\widehat{H}_{F I R T}-H$ for the FIRT estimators $\widehat{H}_{F I R T}$ studied in [16]. As in Figure 4 in [15] these Boxplots were computed by using samples of $n=256$ independent replications of the estimators, obtained from 256 independently simulated paths of the LFSM process for an index of stability $\alpha=1.5$ for $M=2^{16}$ points.

Concerning the parameters $A$ and $L$ let us observe that these parameters do not seem to have a significant influence on the estimated results. It is not surprising that $L$ has less influence than $M$ according to the upper bound given by (3.5) obtained in Corollary 3.3. Actually, since $\alpha=1.5$ for all $H \neq 0.9$ we have $1 / 2-(H-1 / \alpha)<$ $1 / 2$. In order to compare our approximation to the ones of [15], we used the same 
index of stability $\alpha=1.5$ and the same number $n=256$ of samples as in Figure 3 (wavelet estimator) and Figure 4 (FIRT estimator) of [15]. Let us point out that their discretization parameters have a greater influence on the wavelet estimator than in our case, which makes the optimal choice for these parameters more difficult. Moreover, as already observed in Figure 2, our standard deviations are really smaller than in [15] and we also obtain better results for the FIRT estimator. For another indication on the quality of our approximation, let us also refer to the bottom panel of Figure 2 in [20] (also obtained for $\alpha=1.5$ ) and observe that our results are more accurate.

\section{Appendix}

Proof of Proposition 3.1. Let

$$
E_{A}(t)=X(t)-Y_{A}(t)=\int_{-\infty}^{-A}\left((t-z)_{+}^{H-1 / \alpha}-(-z)_{+}^{H-1 / \alpha}\right) Z_{\alpha}(d z) .
$$

Then

$$
\left\|E_{A}(t)\right\|_{\alpha}^{\alpha}=\int_{A}^{\infty}\left|(t+z)^{H-1 / \alpha}-z^{H-1 / \alpha}\right|^{\alpha} d z .
$$

Assume first that $0 \leq t \leq T$. Then, since $1 / \alpha<H<1$, by the mean value theorem $(t+z)^{H-1 / \alpha}-z^{H-1 / \alpha} \leq(H-1 / \alpha) z^{H-1-1 / \alpha} t$ and hence

$$
\left\|E_{A}(t)\right\|_{\alpha}^{\alpha} \leq(H-1 / \alpha)^{\alpha} t^{\alpha} \int_{A}^{\infty} z^{\alpha H-1-\alpha} d z \leq \frac{(H-1 / \alpha)^{\alpha} T^{\alpha}}{\alpha(1-H)} A^{\alpha(H-1)} .
$$

On the other hand, if $-T \leq t<0$ we have $\left|(t+z)^{H-1 / \alpha}-z^{H-1 / \alpha}\right|=z^{H-1 / \alpha}-(t+$ $z)^{H-1 / \alpha} \leq(H-1 / \alpha)(-t)(t+z)^{H-1 / \alpha-1}$ and we get

$$
\left\|E_{A}(t)\right\|_{\alpha}^{\alpha} \leq(H-1 / \alpha)^{\alpha}(-t)^{\alpha} \int_{A}^{\infty}(t+z)^{\alpha H-1-\alpha} d z \leq \frac{(H-1 / \alpha)^{\alpha} T^{\alpha}}{\alpha(1-H)}(A-T)^{\alpha(H-1)}
$$

and the assertion follows. By a similar computation one gets the same bounds for $a=0$ and $b=1$. Since the symmetric case $\varphi(z)=|z|^{H-1 / \alpha}$ is the sum of these two cases we obtain the constant $C_{1}(H, \alpha)$ to hold in either case.

In order to prove Theorem 3.2 we need some estimates on the Fourier-transforms of the $2 A$-periodic functions $\rho_{t}$ and $\varphi$ to control the rate of convergence of their Fourier series.

Lemma 5.1. Assume $1<\alpha \leq 2$ and $H>1 / \alpha$. 
(a) If $\varphi(z)=(-z)_{+}^{H-1 / \alpha}$ (or if $\varphi(z)=(-z)_{-}^{H-1 / \alpha}$ ) then there exists a constant $C_{2}(H, \alpha)$ such that for all $k \in \mathbb{Z} \backslash\{0\}$ and any $t \in \mathbb{R}$ we have

$$
\begin{aligned}
& |\hat{\varphi}(k)| \leq C_{2}(H, \alpha) A^{H-1 / \alpha}|k|^{-1} \\
& \left|\hat{\rho}_{t}(k)\right| \leq 2 C_{2}(H, \alpha) A^{H-1 / \alpha}|k|^{-1},
\end{aligned}
$$

where $C_{2}(H, \alpha)=2+H-1 / \alpha$.

(b) If $\varphi(z)=|z|^{H-1 / \alpha}$ then there exists a constant $C_{2}(H, \alpha)$ such that for all $k \in \mathbb{Z} \backslash\{0\}$ and any $t \in \mathbb{R}$ we have

$$
\begin{aligned}
|\hat{\varphi}(k)| & \leq C_{2}(H, \alpha) A^{H-1 / \alpha}|k|^{-1-H+1 / \alpha} \\
\left|\hat{\rho}_{t}(k)\right| & \leq 2 C_{2}(H, \alpha) A^{H-1 / \alpha}|k|^{-1-H+1 / \alpha},
\end{aligned}
$$

where $C_{2}(H, \alpha)=1+(H-1 / \alpha)$.

Proof. Since $\hat{\rho}_{t}(k)=\left(e^{-i \pi \frac{k}{A} t}-1\right) \hat{\varphi}(k)$ we only consider $\hat{\varphi}(k)$. Moreover, since $\hat{\varphi}(-k)=\overline{\hat{\varphi}(k)}$ it suffices to assume $k \geq 1$. We first consider $\varphi(z)=(-z)_{+}^{H-1 / \alpha}$. Then

$$
\begin{aligned}
\hat{\varphi}(k) & =\frac{1}{2 A} \int_{0}^{A} z^{H-1 / \alpha} e^{i \pi \frac{k}{A} z} d z \\
& =\frac{1}{2} A^{H-1 / \alpha} k^{-1-H+1 / \alpha}\left[\int_{0}^{k} v^{H-1 / \alpha} \cos (\pi v) d v+i \int_{0}^{k} v^{H-1 / \alpha} \sin (\pi v) d v\right]
\end{aligned}
$$

To analyze the first integral on the right hand side of (5.1) we decompose

$$
\begin{aligned}
\int_{0}^{k} v^{H-1 / \alpha} \cos (\pi v) d v & =\int_{0}^{1} v^{H-1 / \alpha} \cos (\pi v) d v+\int_{1}^{k} v^{H-1 / \alpha} \cos (\pi v) d v \\
& =C_{1}+\int_{1}^{k} v^{H-1 / \alpha} \cos (\pi v) d v
\end{aligned}
$$

Integrate by parts twice to obtain

$$
\begin{aligned}
\int_{1}^{k} v^{H-1 / \alpha} \cos (\pi v) d v= & -\frac{H-1 / \alpha}{\pi} \int_{1}^{k} v^{H-1 / \alpha-1} \sin (\pi v) d v \\
= & \frac{H-1 / \alpha}{\pi^{2}}\left((-1)^{k} k^{H-1 / \alpha-1}-1\right) \\
& +\frac{(H-1 / \alpha)(1-H+1 / \alpha)}{\pi^{2}} \int_{1}^{k} v^{H-1 / \alpha-2} \cos (\pi v) d v .
\end{aligned}
$$

Since

$$
\frac{(H-1 / \alpha)(1-H+1 / \alpha)}{\pi^{2}}\left|\int_{1}^{k} v^{H-1 / \alpha-2} \cos (\pi v) d v\right| \leq \frac{H-1 / \alpha}{\pi^{2}}\left(1-k^{H-1 / \alpha-1}\right)
$$


we obtain

$$
\begin{aligned}
\left|\int_{0}^{k} v^{H-1 / \alpha} \cos (\pi v) d v\right| & \leq\left|C_{1}\right|+\left|\int_{1}^{k} v^{H-1 / \alpha} \cos (\pi v) d v\right| \\
& \leq 1+2 \frac{H-1 / \alpha}{\pi^{2}}
\end{aligned}
$$

Similarly we have

$$
\int_{0}^{k} v^{H-1 / \alpha} \sin (\pi v) d v=\int_{0}^{1} v^{H-1 / \alpha} \sin (\pi v) d v+\int_{1}^{k} v^{H-1 / \alpha} \sin (\pi v) d v .
$$

Again, using integration by parts we conclude

$$
\begin{aligned}
& \int_{1}^{k} v^{H-1 / \alpha} \sin (\pi v) d v \\
& \quad=\frac{(-1)^{k+1} k^{H-1 / \alpha}-1}{\pi}+\frac{(H-1 / \alpha)(1-H+1 / \alpha)}{\pi^{2}} \int_{1}^{k} v^{H-1 / \alpha-2} \sin (\pi v) d v
\end{aligned}
$$

and hence

$$
\begin{aligned}
\left|\int_{0}^{k} v^{H-1 / \alpha} \sin (\pi v) d v\right| & \leq 1+\left|\int_{1}^{k} v^{H-1 / \alpha} \sin (\pi v) d v\right| \\
& \leq 1+\frac{1+k^{H-1 / \alpha}}{\pi}+\frac{H-1 / \alpha}{\pi^{2}} .
\end{aligned}
$$

Now (5.1) together with (5.2) and (5.3) imply part (a) of the Lemma.

For the proof of part (b) note that if $\varphi(z)=|z|^{H-1 / \alpha}$ then

$$
\hat{\varphi}(k)=A^{H-1 / \alpha} k^{-1-H+1 / \alpha} \int_{0}^{k} v^{H-1 / \alpha} \cos (\pi v) d v
$$

and hence the assertion follows from (5.2).

Proof of Theorem 3.2. Let $1<\alpha \leq 2$ and $1 / \alpha<H<1$. Then,

$$
\begin{aligned}
\left\|Y_{A}(t)-Y_{A, M, L}(t)\right\|_{\alpha} & =\left\|\int_{-A}^{A}\left[\rho_{t}(y)-\sum_{j=-L}^{L-1} 1_{\left[z_{j}, z_{j+1}[\right.}(y)\left(D_{A, M} * \rho_{t}\right)\left(z_{j}\right)\right] Z_{\alpha}(d y)\right\|_{\alpha} \\
& =\left(\int_{-A}^{A}\left|\rho_{t}(y)-\sum_{j=-L}^{L-1} 1_{\left[z_{j}, z_{j+1}[\right.}(y)\left(D_{A, M} * \rho_{t}\right)\left(z_{j}\right)\right|^{\alpha} d y\right)^{1 / \alpha} .
\end{aligned}
$$

Since $\alpha>1$, we can write

$$
\rho_{t}=\sum_{|k| \leq M} \hat{\rho}_{t}(k) e_{k}+\sum_{|k|>M} \hat{\rho}_{t}(k) e_{k}=D_{A, M} * \rho_{t}+\sum_{|k|>M} \hat{\rho}_{t}(k) e_{k},
$$


where the series converges in $L^{\alpha}$. Therefore, by Minkowski's inequality we obtain

$$
\begin{aligned}
& \left\|Y_{A}(t)-Y_{A, M, L}(t)\right\|_{\alpha} \leq\left(\int_{-A}^{A}\left|\sum_{|k|>M} \hat{\rho}_{t}(k) e_{k}(y)\right|^{\alpha} d y\right)^{1 / \alpha} \\
+ & \left(\int_{-A}^{A}\left|D_{A, M} * \rho_{t}(y)-\sum_{j=-L}^{L-1} 1_{\left[z_{j}, z_{j+1}[\right.}(y)\left(D_{A, M} * \rho_{t}\right)\left(z_{j}\right)\right|^{\alpha} d y\right)^{1 / \alpha} \\
= & V(A, M)+\left(\int_{-A}^{A}\left|\sum_{j=-L}^{L-1} 1_{\left[z_{j}, z_{j+1}[\right.}(y)\left[D_{A, M} * \rho_{t}(y)-D_{A, M} * \rho_{t}\left(z_{j}\right)\right]\right|^{\alpha} d y\right)^{1 / \alpha} \\
= & V(A, M)+U(A, M, L) .
\end{aligned}
$$

We first bound $V(A, M)$. Observe that by Hölder's inequality with $p=2 / \alpha$ and $q^{-1}=1-\alpha / 2$, for $f \in L^{2}([-A, A], d y) \subset L^{\alpha}([-A, A], d y)$,

$$
\left(\int_{-A}^{A}|f(y)|^{\alpha} d y\right)^{1 / \alpha} \leq(2 A)^{(1 / \alpha)-(1 / 2)}\left(\int_{-A}^{A}|f(y)|^{2} d y\right)^{1 / 2} .
$$

Hence, combined with the Plancherel-identity we get

$$
\begin{aligned}
V(A, M) & =\left(\int_{-A}^{A}\left|\sum_{|k|>M} \hat{\rho}_{t}(k) e_{k}(y)\right|^{\alpha} d y\right)^{1 / \alpha} \\
& \leq(2 A)^{(1 / \alpha)-(1 / 2)}\left(\int_{-A}^{A}\left|\sum_{|k|>M} \hat{\rho}_{t}(k) e_{k}(y)\right|^{2} d y\right)^{1 / 2} \\
& =(2 A)^{1 / \alpha}\left(\frac{1}{2 A} \int_{-A}^{A}\left|\sum_{|k|>M} \hat{\rho}_{t}(k) e_{k}(y)\right|^{2} d y\right)^{1 / 2} \\
& =(2 A)^{1 / \alpha}\left(\sum_{|k|>M}\left|\hat{\rho}_{t}(k)\right|^{2}\right)^{1 / 2}
\end{aligned}
$$

In the case $\varphi(z)=(-z)_{+}^{H-1 / \alpha}$, using Lemma 5.1(a),

$$
\begin{aligned}
V(A, M) & \leq 2^{1+1 / \alpha} C_{2}(H, \alpha) A^{H}\left(\sum_{|k|>M}|k|^{-2}\right)^{1 / 2} \\
& \leq 2^{3 / 2+1 / \alpha} C_{2}(H, \alpha)\left(\int_{M}^{\infty} x^{-2} d x\right)^{1 / 2} \\
& =C_{3}(H, \alpha) A^{H} M^{-1 / 2}
\end{aligned}
$$


where $C_{3}(H, \alpha)=2^{3 / 2+1 / \alpha}(2+H-1 / \alpha)$. Similarly, if $\varphi(z)=|z|^{H-1 / \alpha}$ we get using Lemma 5.1(b) that

$$
V(A, M) \leq C_{3}(H, \alpha) A^{H} M^{-1 / 2-(H-1 / \alpha)}
$$

where now $C_{3}(H, \alpha)=2^{3 / 2+1 / \alpha}(1+2 H-2 / \alpha)^{-1 / 2}(1+H-1 / \alpha)$.

Moreover, by Minkowski's inequality

$$
U(A, M, L) \leq \sum_{j=-L}^{L-1}\left(\int_{z_{j}}^{z_{j+1}}\left|D_{A, M} * \rho_{t}(y)-D_{A, M} * \rho_{t}\left(z_{j}\right)\right|^{\alpha} d y\right)^{1 / \alpha} .
$$

Observe further that

$$
D_{A, M} * \rho_{t}(y)-D_{A, M} * \rho_{t}\left(z_{j}\right)=\frac{1}{2 A} \int_{-A}^{A} D_{A, M}(z)\left[\rho_{t}(y-z)-\rho_{t}\left(z_{j}-z\right)\right] d z .
$$

Hence, by Minkowski's integral inequality (see e.g. [13], p.177) we obtain

$$
\begin{aligned}
& \left(\int_{z_{j}}^{z_{j+1}}\left|\frac{1}{2 A} \int_{-A}^{A} D_{A, M}(z)\left[\rho_{t}(y-z)-\rho_{t}\left(z_{j}-z\right)\right] d z\right|^{\alpha} d y\right)^{1 / \alpha} \\
\leq & \frac{1}{2 A} \int_{-A}^{A}\left|D_{A, M}(z)\right|\left(\int_{z_{j}}^{z_{j+1}}\left|\rho_{t}(y-z)-\rho_{t}\left(z_{j}-z\right)\right|^{\alpha} d y\right)^{1 / \alpha} d z .
\end{aligned}
$$

Therefore we have to estimate

$$
U(A, M, L) \leq \frac{1}{2 A} \int_{-A}^{A}\left|D_{A, M}(z)\right| \sum_{j=-L}^{L-1}\left(\int_{z_{j}}^{z_{j+1}}\left|\rho_{t}(y-z)-\rho_{t}\left(z_{j}-z\right)\right|^{\alpha} d y\right)^{1 / \alpha} d z
$$

Note that $\rho_{t}(y-z)-\rho_{t}\left(z_{j}-z\right)=\varphi(t-(y-z))-\varphi\left(t-\left(z_{j}-z\right)\right)-\left(\varphi(y-z)-\varphi\left(z_{j}-z\right)\right)$. Let us denote $I(\varphi, L, z)=\sum_{j=-L}^{L-1}\left(\int_{z_{j}}^{z_{j+1}}\left|\varphi(y-z)-\varphi\left(z_{j}-z\right)\right|^{\alpha} d y\right)^{1 / \alpha}$ and remark that

$$
\sum_{j=-L}^{L-1}\left(\int_{z_{j}}^{z_{j+1}}\left|\rho_{t}(y-z)-\rho_{t}\left(z_{j}-z\right)\right|^{\alpha} d y\right)^{1 / \alpha} \leq I(\check{\varphi}, L, z+t)+I(\varphi, L, z),
$$

where $\check{\varphi}(y)=\varphi(-y)$.

Note that if $\varphi(z)=(-z)_{+}^{H-1 / \alpha}\left(\right.$ or $\left.\varphi(z)=(-z)_{-}^{H-1 / \alpha}\right)$ some of the summands in $I(\check{\varphi}, L, z+t)$ and $I(\varphi, L, z)$ are zero. Moreover, since the estimates in these two cases as well as the symmetric case $\varphi(z)=|z|^{H-1 / \alpha}$ are quite similar, we will only consider the symmetric case and leave the details of the other cases to the reader.

Since $\varphi$ is $2 A$ periodic, $I(\varphi, L, \cdot)$ is also $2 A$ periodic and we just have to analyze $J(L, z)=I(\varphi, L, z)$ for $-A \leq z<A$. Let us denote $j_{0}=j_{0}(z)=[(L / A) z]$ where 
$[v]$ denotes the largest integer less or equal to $v$. Then $-L \leq j_{0} \leq L-1$ and we decompose further $J(L, z)$ (setting $\sum_{j=a}^{b}=0$ if $b<a$ ) as

$$
\begin{aligned}
& J(L, z) \\
= & \sum_{j=-L}^{j_{0}-1}\left(\int_{z_{j}}^{z_{j+1}}\left|\varphi(y-z)-\varphi\left(z_{j}-z\right)\right|^{\alpha} d y\right)^{1 / \alpha} \\
+ & \left(\int_{z_{j_{0}}}^{z_{j_{0}+1}}\left|\varphi(y-z)-\varphi\left(z_{j_{0}}-z\right)\right|^{\alpha} d y\right)^{1 / \alpha}+\left(\int_{z_{j_{0}+1}}^{z_{j_{0}+2}}\left|\varphi(y-z)-\varphi\left(z_{j_{0}+1}-z\right)\right|^{\alpha} d y\right)^{1 / \alpha} \\
+ & \sum_{j=j_{0}+2}^{L-1}\left(\int_{z_{j}}^{z_{j+1}}\left|\varphi(y-z)-\varphi\left(z_{j}-z\right)\right|^{\alpha} d y\right)^{1 / \alpha} \\
= & I_{1}(z)+I_{2}(z)+I_{3}(z)+I_{4}(z) .
\end{aligned}
$$

Observe that if $j \leq j_{0}-1$ we have $z_{j}<z_{j+1} \leq z_{j_{0}} \leq z$ and hence for $z_{j} \leq y \leq z_{j+1}$ we have $z_{j}-z \leq y-z \leq z_{j+1}-z \leq 0$. Since $H<1$ we have $H-1-1 / \alpha<0$ and by the mean value theorem we get in the present case that $\left|\varphi(y-z)-\varphi\left(z_{j}-z\right)\right|=\left(z-z_{j}\right)^{H-1 / \alpha}-(z-y)^{H-1 / \alpha} \leq(H-1 / \alpha)\left(z-z_{j+1}\right)^{H-1-1 / \alpha}\left(y-z_{j}\right)$.

This implies

$$
\begin{aligned}
I_{1}(z) & =\sum_{j=-L}^{j_{0}-1}\left(\int_{z_{j}}^{z_{j+1}}\left|\varphi\left(z_{j}-z\right)-\varphi(y-z)\right|^{\alpha} d y\right)^{1 / \alpha} \\
& \leq(H-1 / \alpha) \sum_{j=-L}^{j_{0}-1}\left(z-z_{j+1}\right)^{H-1-1 / \alpha}\left(\int_{z_{j}}^{z_{j+1}}\left(y-z_{j}\right)^{\alpha} d y\right)^{1 / \alpha} \\
& \leq(H-1 / \alpha)(\alpha+1)^{-1 / \alpha}\left(\frac{A}{L}\right)^{1+1 / \alpha} \sum_{j=-L}^{j_{0}-1}\left(z-z_{j}\right)^{H-1-1 / \alpha} \\
& \leq 2(H-1 / \alpha)(\alpha+1)^{-1 / \alpha}\left(\frac{A}{L}\right)^{1+1 / \alpha} \int_{-L}^{(L / A) z}(z-x(A / L))^{H-1-1 / \alpha} d x \\
& =2(\alpha+1)^{-1 / \alpha}\left(\frac{A}{L}\right)^{1+1 / \alpha} \frac{L}{A}(z+A)^{H-1 / \alpha} \leq 2 \cdot 2^{H-1 / \alpha}(\alpha+1)^{-1 / \alpha} A^{H} L^{-1 / \alpha} .
\end{aligned}
$$

Since $H-1 / \alpha \in(0,1)$ the function $\varphi$ is Hölder of order $H-1 / \alpha$ on $[-A, A)$ with $|\varphi(x)-\varphi(y)| \leq|x-y|^{H-1 / \alpha}$ for all $-A \leq x, y<A$. Since $z \in\left[z_{j_{0}}, z_{j_{0}+1}\right)$

$$
I_{2}(z)=\left(\int_{z_{j_{0}}}^{z_{j_{0}+1}}\left|\varphi(y-z)-\varphi\left(z_{j_{0}}-z\right)\right|^{\alpha} d y\right)^{1 / \alpha} \leq(A / L)^{H} .
$$


Similarly $I_{3}(z) \leq(A / L)^{H}$. Moreover, since $H>1 / \alpha$ we get $I_{2}(z)+I_{3}(z) \leq 2 A^{H} L^{-1 / \alpha}$. For $I_{4}(z)$, observe that if $j \geq j_{0}+1$ we have $z_{j} \geq z_{j_{0}+1}>z$ so for $z_{j} \leq y \leq z_{j+1}$ we have using the mean value theorem again

$\left|\varphi(y-z)-\varphi\left(z_{j}-z\right)\right|=(y-z)^{H-1 / \alpha}-\left(z_{j}-z\right)^{H-1 / \alpha} \leq(H-1 / \alpha)\left(z_{j}-z\right)^{H-1-1 / \alpha}\left(y-z_{j}\right)$.

This implies

$$
\begin{aligned}
I_{4}(z) & =\sum_{j=j_{0}+2}^{L-1}\left(\int_{z_{j}}^{z_{j+1}}\left|\varphi\left(z_{j}-z\right)-\varphi(y-z)\right|^{\alpha} d y\right)^{1 / \alpha} \\
& \leq(H-1 / \alpha) \sum_{j=j_{0}+2}^{L-1}\left(z_{j}-z\right)^{H-1-1 / \alpha}\left(\int_{z_{j}}^{z_{j+1}}\left(y-z_{j}\right)^{\alpha} d y\right)^{1 / \alpha} \\
& \leq(H-1 / \alpha)(\alpha+1)^{-1 / \alpha}\left(\frac{A}{L}\right)^{1+1 / \alpha} \sum_{j=j_{0}+2}^{L-1}\left(z_{j}-z\right)^{H-1-1 / \alpha} \\
& \left.\leq 2(H-1 / \alpha)(\alpha+1)^{-1 / \alpha}\left(\frac{A}{L}\right)^{1+1 / \alpha} \int_{(L / A) z}^{L-1}((A / L) x-z)\right)^{H-1-1 / \alpha} d x \\
& =2(\alpha+1)^{-1 / \alpha}\left(\frac{A}{L}\right)^{1+1 / \alpha} \frac{L}{A}(A-z)^{H-1 / \alpha} \leq 2 \cdot 2^{H-1 / \alpha}(\alpha+1)^{-1 / \alpha} A^{H} L^{-1 / \alpha}
\end{aligned}
$$

Therefore we have for $-A \leq z \leq A$ that

$$
J(L, z) \leq 2\left(2^{1+H-1 / \alpha}(\alpha+1)^{-1 / \alpha}+1\right) A^{H} L^{-1 / \alpha}=C_{5}(H, \alpha) A^{H} L^{-1 / \alpha} .
$$

Finally, in view of (5.5) we get

$$
U(A, M, L) \leq 2 C_{5}(H, \alpha) A^{H} L^{-1 / \alpha} \frac{1}{2 A} \int_{-A}^{A}\left|D_{A, M}(z)\right| d z
$$

Note further that we have

$$
\begin{aligned}
\frac{1}{2 A} \int_{-A}^{A}\left|D_{A, M}(x)\right| d x & =\frac{1}{\pi} \int_{-\frac{2 M+1}{2} \pi}^{\frac{2 M+1}{2} \pi} \frac{|\sin (x)|}{\mid(2 M+1) \sin (x /(2 M+1) \mid} d x \\
& =\frac{1}{\pi} \int_{-\frac{2 M+1}{2} \pi}^{\frac{2 M+1}{2} \pi} \frac{|\sin (x)|}{|x|}\left|\frac{x /(2 M+1)}{\sin (x /(2 M+1)}\right| d x \\
& \leq \frac{1}{2} \int_{-\frac{2 M+1}{2} \pi}^{\frac{2 M+1}{2} \pi} \frac{|\sin (x)|}{|x|} d x
\end{aligned}
$$


using the fact that $|\sin (x)| \geq \frac{2}{\pi}|x|$ for $|x| \leq \frac{\pi}{2}$. Moreover,

$$
\begin{aligned}
\frac{1}{2} \int_{-\frac{2 M+1}{2} \pi}^{\frac{2 M+1}{2} \pi} \frac{|\sin (x)|}{|x|} d x & \leq \frac{1}{2} \int_{-(M+1) \pi}^{(M+1) \pi} \frac{|\sin (x)|}{|x|} d x=\sum_{k=0}^{M} \int_{0}^{\pi} \frac{\sin (x)}{x+k \pi} d x \\
& \leq \pi+\frac{2}{\pi}(1+\log (M)) .
\end{aligned}
$$

Hence, $\frac{1}{2 A} \int_{-A}^{A}\left|D_{A, M}(x)\right| d x \leq 2 \log (M)$, for $M \geq e^{4}$, and

$$
U(A, M, L) \leq 4 C_{5}(H, \alpha) A^{H} L^{-1 / \alpha} \log (M)=C_{4}(H, \alpha) A^{H} L^{-1 / \alpha} \log (M),
$$

which concludes the proof.

ACKNOWLEDGEment. The authors would like to thank various referees of an earlier version of this paper for suggesting numerous improvements.

\section{REFERENCES}

[1] J. M. Chambers, C. Mallows and B. W. Stuck (1976) A method for simulating stable random variables. J. American Stat. Assoc. 71(354), 340-344. with a correction in J. American Stat. Assoc. 82(398), 704.

[2] S. Cohen, C. Lacaux and M. Ledoux (2006) A general framework for simulation of fractional fields. Preprint available at http://www.iecn.u-nancy.fr/ lacaux/FICHIERS/ Simu-fractional-fields.pdf

[3] C. R. Dietrich and G. N. Newsam (1997) Fast and exact simulation of stationary Gaussian processes through circulant embedding of the covariance matrix. SIAM J. Sci. Comput. 18(4), $1088-1107$.

[4] P. Embrechts and M. Maejima (2002) Self-similar Processes. Princeton University Press.

[5] P. Doukhan, G. Oppenheim and M.S. Taqqu (2003) Theory and Applications of Long-Range Dependence. Birkhäuser, Bosten.

[6] D. Greenspan (1971) Introduction to Numerical Analysis and Applications. Markham Publishing Company, Chicago.

[7] O. Kallenberg (2002) Foundations of Modern Probability. Springer, New York.

[8] Y. Katznelson (1976) An introduction to Harmonic Analysis. Dover Publ. Inc., New York.

[9] W.E. Leland, M.S. Taqqu, W. Willinger and D.V. Wilson (1994) On the self-similar nature of Ethernet traffic. IEEE/ACM Transactions on Networking 2, 1-15.

[10] N. Kôno and M. Maejima (1991) Hölder continuity of sample path of some self-similar stable processes. Tokyo J. Math. 14, 93-100.

[11] K. Park and W. Willinger, eds. (2000) Self-Similar Network Traffic and Performance Evaluation, J. Wiley and Sons, Inc., New York.

[12] E. Perrin, R. Harba, R. Jennane and I. Iribarren (2002) Fast and Exact Synthesis for 1-D Fractional Brownian Motion and Fractional Gaussian Noises. IEEE Signal Processing Letters 9(11), 382-384.

[13] W. Rudin (1987) Real and Complex Analysis. McGRAW-HILL, New York.

[14] G. Samorodnitsky and M. S. Taqqu (1994) Stable non-Gaussian Random Processes. Chapman and Hall, New York. 
[15] S. Stoev and M. S. Taqqu (2004) Simulation methods for linear fractional stable motion and FARIMA using the Fast Fourier Transform. Fractals 12(1), 95-121.

[16] S. Stoev, V. Pipiras and M. S. Taqqu (2002) Estimation of the Self-similarity Parameter in Linear Fractional Stable Motion. Signal Process. 82, 1873-1901.

[17] K. Takashima (1989) Sample path properties of ergodic self-similar processes. Osaka J. of Math. 26, 159-189.

[18] W. Willinger, V. Paxson and M. S. Taqqu (1998) Self-similarity and heavy tails: structural modeling of network traffic. in R. Adler, R. Feldman and M. Taqqu, eds. A Practical Guide to Heavy Tails: Statistical Techniques and Applications, Birkhäuser, Bosten, 27-53.

[19] W.B. Wu, G. Michailidis and D. Zhang (2004) Simulating sample path of linear fractional stable motion. IEEE Transactions on Information Theory 50(6), 1086-1096.

[20] W. B. Wu, G. Michailidis and D. Zhang (2004) An efficient method for simulating fractional stable motion. Simulation Conference, 2002. Proceedings of the Winter 2, 1958-1963.

Hermine Biermé, MAP5 Université René Descartes, 45 Rue des Saints Pères, 75270 PARIS CEDEX 06, FRANCE

E-mail address: hermine.bierme@math-info.univ-paris5.fr

Hans-Peter Scheffler, FB 6 Mathematik, Universität Siegen, D-57068 Siegen, GerMANY

E-mail address: scheffler@mathematik.uni-siegen.de 